Formatted for: Experimental Brain Research

\title{
Tilt and Translation Motion Perception During Off-Vertical Axis Rotation
}

\author{
Scott J. Wood ${ }^{1,2}$, Millard F. Reschke ${ }^{2}$, Gilles Clément ${ }^{3}$ \\ ${ }^{1}$ Universities Space Research Association, Houston, TX, USA \\ ${ }^{2}$ Neuroscience Laboratory, NASA Johnson Space Center, Houston, TX, USA \\ ${ }^{3}$ Centre de Recherche Cerveau et Cognition, UMR 5549 CNRS/UPS, Toulouse, France
}

Running title: Motion perception during OVAR

\section{Address for Correspondence:}

Scott J. Wood, Ph.D.

NASA JSC, Mail Code SK-272

Tel (281) 483-7294

2101 NASA Parkway

Fax (281) 244-5734

Houston, TX 77058

Email: sjwood@ems.jsc.nasa.gov

Acknowledgements: Philippe Tauzin of Service Commun Multimedia at the University Paul Sabatier in Toulouse provided the artwork. We thank Pierre Denise for his contributions to the questions used for verbal reports of self-motion perception. This research was funded by NASA (DSO 499) and Centre National d'Etudes Spatiales. 


\section{ABSTRACT}

The effect of stimulus frequency on tilt and translation motion perception was studied during constant velocity off-vertical axis rotation (OVAR), and compared to the effect of stimulus frequency on eye movements. Fourteen healthy subjects were rotated in darkness about their longitudinal axis $10^{\circ}$ and $20^{\circ}$ off-vertical at $0.125 \mathrm{~Hz}$, and $20^{\circ}$ off-vertical at $0.5 \mathrm{~Hz}$. Oculomotor responses were recorded using videography, and perceived motion was evaluated using verbal reports and a joystick with four degrees of freedom (pitch and roll tilt, medial-lateral and anterior-posterior translation). During the lower frequency OVAR, subjects reported the perception of progressing along the edge of a cone. During higher frequency OVAR, subjects reported the perception of progressing along the edge of an upright cylinder. The modulation of both tilt recorded from the joystick and ocular torsion significantly increased as the tilt angle increased from $10^{\circ}$ to $20^{\circ}$ at $0.125 \mathrm{~Hz}$, and then decreased at $0.5 \mathrm{~Hz}$. Both tilt perception and torsion slightly lagged head orientation at $0.125 \mathrm{~Hz}$. The phase lag of torsion increased at $0.5 \mathrm{~Hz}$, while the phase of tilt perception did not change as a function of frequency. The amplitude of both translation perception recorded from the joystick and horizontal eye movements was negligible at $0.125 \mathrm{~Hz}$ and increased as a function of stimulus frequency. While the phase lead of horizontal eye movements decreased at $0.5 \mathrm{~Hz}$, the phase of translation perception did not vary with stimulus frequency and was similar to the phase of tilt perception during all conditions. During dynamic linear acceleration in the absence of other sensory input (canal, vision) a change in stimulus frequency alone elicits similar changes in the amplitude of both self motion perception and eye movements. However, in contrast to the eye movements, the phase of both perceived tilt and translation motion is not altered by stimulus frequency. We conclude that the neural processing to distinguish tilt and translation linear acceleration stimuli differs between eye movements and motion perception.

Keywords: OVAR, otolith, acceleration, orientation, VOR 


\section{INTRODUCTION}

The otolith organs of the vestibular system transduce linear acceleration from both translation motion and head tilt relative to gravity. The ambiguity between these two types of linear acceleration must be resolved for both the accurate perception of motion and generation of compensatory eye movements during different types of head movement (Mayne 1974). Constant velocity rotation of head and body around an axis tilted with respect to gravity (Off-Vertical Axis Rotation, OVAR) is one means of providing a dynamic linear acceleration stimulus. During OVAR, the angle of tilt determines the amplitude of the linear acceleration stimulus, whereas the velocity of rotation determines its frequency content. The sinusoidally varying linear acceleration during OVAR in darkness elicits a modulation of horizontal, torsional and vertical eye movements (Guedry 1965; Benson and Bodin 1966; Darlot et al. 1988; Haslwanter et al. 2000; Yagi et al. 2000). Recent work in our laboratory has demonstrated that lower frequency responses $(<0.3 \mathrm{~Hz})$ during OVAR are principally characterized by the modulation of tiltposition dependent ocular reflexes (e.g., torsion) whereas the modulation of translational ocular reflexes (e.g., horizontal) are predominant at higher frequencies (Wood 2002).

Although motion perception has been studied during OVAR (Guedry 1965; Graybiel and Miller 1970; Denise et al. 1988), these studies have typically employed lower frequency stimuli below $0.3 \mathrm{~Hz}$. The motion perception commonly reported during OVAR at these low frequencies is a progression along the circumferential edge of a downward oriented cone (Denise et al. 1988). The angle of the perceived conical body path increases with the actual tilt angle of the rotation axis, indicating that the perception process depends on inputs signaling head and body orientation with respect to gravity. Few studies have been performed using OVAR stimuli greater than $0.3 \mathrm{~Hz}$. In one study, Miller and Graybiel (1973) found that subjects reported "being at or near upright" when rotating at $0.66 \mathrm{~Hz}(240 \%)$. Unfortunately, it was unclear from Miller and Graybiel's report whether this reduced sense of tilt motion at higher frequencies was accompanied by an increased sense of translation analogous to the transition from tilt to translation ocular reflexes (Wood 2002).

The purpose of this research was therefore to examine the effect of stimulus frequency on the perception of motion during OVAR. Specifically, we compared the sensation of whole body tilt versus whole body translation at frequencies above and below the crossover range of tilt and 
translation ocular reflexes $(\sim 0.3 \mathrm{~Hz}$, Wood 2002). The emphasis on whole-body motion is important to provide insight into how the central nervous system is resolving the ambiguity between tilt and translation. The perception of a downward oriented cone described in earlier studies (Denise et al. 1988) involves translation along the edge of the cone, although clearly this results from the perception that one is tilting about an axis below the head. It is important therefore to distinguish translation of the head associated with tilt about an eccentric axis from the perception of whole body translation.

We predicted that the sensation of progressing along a cone reported during lowfrequency OVAR would be replaced by a more pronounced sense of medial-lateral and anteriorposterior translation similar to progression along a cylindrical path at high frequency. In addition to comparing responses at low $(0.125 \mathrm{~Hz})$ and high $(0.5 \mathrm{~Hz})$ frequencies during OVAR, responses were obtained at two angles of tilt $\left(10\right.$ and $\left.20^{\circ}\right)$ in order to compare responses to different magnitudes of linear acceleration. These small angles of tilt were chosen to be within the range of natural behavior. We introduce a new technique for simultaneous recording of perception of both tilt and translation that reflects how the central nervous system distinguishes these two types of linear acceleration for motion perception.

\section{MATERIALS AND METHODS}

\section{$\underline{\text { Subjects }}$}

Fourteen subjects ( 7 females, 7 males), ranging in age from 24-51 yrs (mean 34 yrs) participated in this study. All subjects were required to pass a medical examination, including an audiogram, and have no history of vestibular or oculomotor abnormalities. Normal vestibular (canal) function was also confirmed by post-rotatory responses to velocity step profiles obtained following each experiment trial (gain $0.41 \pm 0.04$, time constant $12.5 \pm 2.0 \mathrm{~s}$ ). The experiment was under taken with the understanding and written consent of each subject. The test procedures were approved by and in compliance with the standards of the NASA institutional review board

for human testing and were performed in accordance with the ethical standards laid down in the 1964 Declaration of Helsinki. 


\section{Equipment}

Subjects were rotated about their longitudinal axis in the Off-Vertical Axis Rotator System at the NASA Johnson Space Center Neurosciences Laboratory. This system utilizes a direct-drive $80 \mathrm{ft}-\mathrm{lb}$ DC motor and high precision tachometer-based servo controller (Neuro Kinetics Inc., Pittsburgh, PA) for motor control and stability during rotation. An electromechanical linear actuator was used to tilt the axis of rotation between upright and $20^{\circ}$ off-vertical. A photo-detector circuit provided once-per-revolution pulses in the nose-up orientation and a tachometer provided rotator velocity.

The rotation was performed in complete darkness, and operator instructions as well as background masking noise were provided via a chair-fixed speaker to minimize any extraneous spatial orientation cues. Subjects were restrained in an upright seated position. The Reid's baseline was approximately in the plane of rotation, with the horizontal canals and utricular macula inclined up approximately $20^{\circ}$ relative to this plane (Curthoys et al. 1977). This orientation was used to maintain a natural head-erect position.

Body support was provided by straps at the shoulders, waist, thighs, knees, and feet. Adjustable pads applied pressure to formable Vac-Pacs ${ }^{\circledR}$ (Olympic Medical, Seattle, WA) to immobilize the mid-torso and uniformly distribute the pressure. The head position was stabilized with adjustable clamps coupled to custom-fitted thermoplastic pads positioned over the forehead and sides of the head with the aid of a skull cap.

Eye movements were recorded on video cassette tapes with a chair-fixed binocular video camera system, which used dichroic mirrors to provide a full field of view $\left(120^{\circ}\right.$ horizontal by $90^{\circ}$ vertical). Small monochrome video cameras were used with near-infrared emitting diodes to allow eye recording in darkness. A timecode was digitally overlaid on each video field $(60 \mathrm{~Hz}$ sample rate) to permit off-line processing and synchronization with the rotation stimuli signals, joystick perception signals and calibration target information. Torsional, horizontal and vertical eye position were processed off-line using an eye tracking system previously described (Wood et al. 1998; Wood 2002).

Pre- and post-test eye measurement calibrations were made by having subjects fixate a series of wall targets positioned $5^{\circ}$ apart over a range of $\pm 25^{\circ}$ horizontally and $\pm 20^{\circ}$ vertically at a distance of $1.7 \mathrm{~m}$. A low-power laser crosshair pointer was mounted to the chair at height of 
the outer canthus and projected toward the calibration array to align the horizontal calibration targets with eye level.

A joystick using four single-axis potentiometers was mounted on the chair directly in front of the subject at mid-torso. Subjects used one hand on the upper stage to indicate the direction and amplitude of perceived body tilt in roll (coronal plane) and pitch (sagittal plane). Subjects used their other hand on the lower stage to indicate direction and amplitude of perceived body translation along medial-lateral (ML) and anterior-posterior (AP) axes (Fig. 1). Subjects were instructed to report 'whole-body' tilt and translation, limiting translation to perceived cylindrical motion path. Given the limited range of the joystick, subjects were instructed to scale their perceived amplitude such that the length of the joystick handle represented one body length. The data were then normalized as a percentage of the maximum peak-to-peak range.

\section{$<$ Insert Figure 1 about here $>$}

\section{$\underline{\text { Procedure }}$}

The experimental protocol included 2 velocities of rotation, $45 \% \mathrm{~s}$ and $180 \% \mathrm{~s}$ corresponding to $0.125 \mathrm{~Hz}$ and $0.5 \mathrm{~Hz}$, respectively, during OVAR at $20^{\circ}$ of tilt. A tilt angle of $10^{\circ}$ was also tested during $0.125 \mathrm{~Hz}$ rotation, which allowed a direct comparison of our motion perception results with those previously obtained by Denise et al. (1988).

Subjects were instructed to keep their eyes open in the dark and look straight ahead at an imaginary horizon. Data collection started with the chair tilted $10^{\circ}$ and the subject in the nose-up position. The chair accelerated at $25 \% \mathrm{~s}^{2}$ up to $45 \%$ s constant velocity. Following $60 \mathrm{~s}$ to allow the primary per-rotatory nystagmus to decay, eye movements were recorded during constant velocity at $0.125 \mathrm{~Hz}$ for $80 \mathrm{~s}$ (i.e., 10 cycles). The subjects were then requested to reproduce with the joystick the direction and amplitude of their perceived motion (Fig. 1). The joystick signals were recorded for a minimum of 10 cycles before the following systematic questions were asked by the operator: (1) do you perceive moving along the edges of a cone and/or cylinder, (2) is the path circular or elliptical, (3) if cone, quantify the amplitude of tilt and location of its apex (tip) relative to the head, (4) quantify the degree to which the long axis of the cone or cylinder is tilted 
relative to vertical, and (5) what direction do you feel you are moving (clockwise, CW or counter-clockwise, $\mathrm{CCW}$, as viewed from above).

The chair was then tilted to $20^{\circ}$ in less than $5 \mathrm{~s}$. After obtaining eye movements (10 cycles), joystick ( 10 cycles) and verbal reports at $0.125 \mathrm{~Hz}$ during $20^{\circ} \mathrm{OVAR}$, the chair was accelerated at $25^{\circ} / \mathrm{s}^{2}$ up to $0.5 \mathrm{~Hz}$ constant velocity. Following $60 \mathrm{~s}$ to allow the primary canal response to decay, eye movements, joystick data and verbal reports were acquired during approximately 15 cycles each. The rotation axis was then brought back to Earth-vertical. At this point none of the subjects perceived rotation, and the chair was stopped (deceleration $25 \% \mathrm{~s}^{2}$ ), thereby eliciting a horizontal post-rotatory nystagmus. After several minutes of rest, rotation in the opposite direction was performed using the same protocol. The direction of rotation (CW or $\mathrm{CCW}$ ) was counterbalanced across subjects. The test was terminated early at the onset of motion sickness (mild nausea) reported by the subjects.

\section{Data Analysis}

Eye position, joystick, and rotator data were filtered with a predictive finite-impulse response (FIR), median hybrid filter (Engelken and Stevens 1990). Conjugate eye position (average of left and right) was differentiated to calculate eye velocity. Fast phase components of the eye movements were identified using acceleration and velocity thresholds and verified manually to be excluded from the analysis.

Nonlinear least squares sinusoidal curve fits were used to describe the modulation of eye and joystick responses as a function of the sinusoidal-varying linear acceleration stimulus. The curve fits were used to determine the amplitude and phase of torsion position, horizontal slowphase velocity (SPV), pitch tilt, roll tilt, ML translation and AP translation. Phases for torsion position and joystick data were expressed relative to tilt position with positive leading and negative lagging. Phases for horizontal SPV were offset by $90^{\circ}$ to account for the fact that they are compensatory for change in head position.

These response parameters were analyzed with repeated measures ANOVAs using a commercial statistics program (JMP, SAS Institute Inc.). Using an alpha error of 0.05 as the decision rule, the null hypothesis that there is no difference across tilt angle and frequency was tested with Wilke's lambda serving as the critical statistic. 


\section{RESULTS}

Seven out of the 14 subjects completed the entire protocol including both directions of rotation at both velocities and both tilt angles (indicated by asterisk in Table 1). For the remaining subjects, the experiment was stopped at the onset of mild nausea. Four subjects did not develop symptoms until the completion of the first run or during the second run in the opposite direction. Three developed symptoms during the first direction of rotation, one at the lower speed of rotation. The modulation of eye movement and joystick data were not significantly different across rotation direction for the seven subjects who completed the entire protocol. Therefore, data from the first run was used for the remainder of the analyses below. Figure 2 shows samples of eye and joystick responses for one typical subject during $20^{\circ} \mathrm{OVAR}$ at both $0.125 \mathrm{~Hz}(\mathrm{~A})$ and $0.5 \mathrm{~Hz}(\mathrm{~B})$.

\section{$<$ Insert Figure 2 about here $>$}

\section{$\underline{\text { Verbal reports }}$}

Verbal reports of the perceived motion path were consistent across $\mathrm{CW}$ and CCW directions of rotation, and are therefore combined in Table 1. During OVAR at $10^{\circ}$ tilt, all subjects reported the pattern of body motion previously described by Denise et al. (1988): progression along a conical path while facing the same direction. For $20^{\circ}$ tilt at $0.125 \mathrm{~Hz}, 79 \%$ of subjects continued to perceive a conical path. The average amplitude of perceived tilt was reported to be $19.1^{\circ}\left( \pm 1.9^{\circ} \mathrm{sem}\right)$ for $10^{\circ} \mathrm{OVAR}$ and $34.4^{\circ}\left( \pm 2.3^{\circ} \mathrm{sem}\right)$ for $20^{\circ} \mathrm{OVAR}$. However, at $0.5 \mathrm{~Hz} 86 \%$ of subjects reported a cylindrical path with no sense of tilt, and the remaining two reported dramatically reduced sense of tilt $\left(5^{\circ}\right)$. At both frequencies and tilt angles, the majority of subjects reported a circular path with the long axis of the cone or cylinder upright.

$<$ Insert Table 1 about here $>$ 
The apex of the cone at $0.125 \mathrm{~Hz}$ was perceived below the head at an average estimated distance of $2.7 \mathrm{~m}( \pm 0.5 \mathrm{~m} \mathrm{sem})$ for $10^{\circ} \mathrm{OVAR}$ and $1.6 \mathrm{~m}( \pm 0.4 \mathrm{~m} \mathrm{sem})$ for $20^{\circ} \mathrm{OVAR}$. It is interesting to note that based on this average distance and tilt amplitude, the radius of the perceived conical path was the same for both tilt angles $(\sim 0.9 \mathrm{~m})$. The subjects generally felt progressing along the cone or cylinder in the opposite direction of the actual spinning.

Eye movement responses

The torsion amplitude doubled from $10^{\circ}$ to $20^{\circ}$ OVAR whereas the phase remained the same across tilt angles at $0.125 \mathrm{~Hz}$ (Figure 3). However; consistent with our previous study (Wood 2002), both amplitude and phase of torsion and horizontal slow phase velocity (SPV) were significantly affected by stimulus frequency. At the high frequency, the torsion amplitude decreased while there was a greater phase lag relative to the low frequency (Figures 2 and 3 ). The amplitude of the horizontal SPV was negligible at both $10^{\circ}$ to $20^{\circ} \mathrm{OVAR}$ at $0.125 \mathrm{~Hz}$, but significantly increased at the high frequency. The horizontal SPV phase was leading the linear stimulus at the low frequency, and became more in phase at the high frequency.

$<$ Insert Figure 3 about here $>$

\section{$\underline{\text { Joystick responses }}$}

The joystick settings were consistent with the perceived motion path verbally reported by the subjects. The amplitude of perceived roll and pitch tilt was larger than perceived ML and AP translation, respectively, at the low frequency for both $10^{\circ}$ and $20^{\circ} \mathrm{OVAR}$ (Figure 4A and 4B). The effect of stimulus frequency on the amplitude of the joystick measures was similar to the effect observed for the eye movement amplitude described above. As the stimulus increased from $0.125 \mathrm{~Hz}$ to $0.5 \mathrm{~Hz}$, the amplitude of tilt significantly decreased while the amplitude of translation increased. In contrast to the eye movements; however, the average phase of the perceived motion was not affected by stimulus frequency (Figure 4C and 4D), consistently lagging the stimulus by $<30^{\circ}$. In fact, the phase of the perceived tilt was not significantly 
different from the phase of the corresponding perceived translation (roll versus ML, pitch versus $\mathrm{AP})$ in any of the tested conditions.

$$
<\text { Insert Figure } 4 \text { about here }>
$$

\section{DISCUSSION}

One major contribution of this study is that, as already shown for eye movements (Wood 2002), the amplitude of both tilt and translation motion perception during OVAR varies as a function of linear acceleration frequency in the absence of visual and canal cues. However; in contrast with eye movements, the phase of motion perception does not vary with stimulus frequency. We infer that the neural processing required to distinguish between tilt and translation differs for eye movements and motion perception (Merfeld et al. 2005). While the tilt and translation ocular reflexes appear to operate in a more independent fashion, the timing of perceived tilt and translation influence each other. We conclude that the perceived motion path during linear acceleration results from a composite representation of tilt and translation. While increasing stimulus frequency results in striking decreases in the amplitude of tilt (Glasauer 1995; Merfeld et al. 2005), the concomitant increase in sense of translation serves to maintain the phase of the perceived motion relative to the stimulus.

Figure 5 illustrates the ambiguous motion paradigm used in the current study. During OVAR a CW rotation results in a sequence of nose-down, left-ear down, nose-up, and right-ear down orientations (Fig. 5A). However, one would need to tilt the head along a $\mathrm{CCW}$ motion path to result in the same sequence of orientations without rotating about a longitudinal axis. This explains why the perceived direction of rotation during OVAR at low velocities is generally opposite to the actual direction of rotation (Graybiel and Miller 1970; Denise et al. 1988). The principal ambiguity during constant velocity OVAR is whether the sinusoidally varying linear acceleration is due to tilt or translation. For example, the maximal leftward interaural acceleration due to gravity occurs in the right-ear-down (RED) orientation. This can be interpreted as either resulting from a rightward roll tilt, or resulting from translation acceleration in the leftward direction. Since acceleration is $180^{\circ}$ out of phase from position, the maximal leftward acceleration during sinusoidal translation occurs when one is at the extreme right 
position. Therefore, at the RED orientation, our subjects reported either the maximal rightward tilt (Fig. 5B) or maximal rightward translation position (Fig. 5C). If one perceives the linear acceleration during OVAR as tilt, the motion path is described as moving along the edge of a cone, while always facing the same direction (Fig. 5B). If one perceives linear acceleration during OVAR as translation, the motion path is moving along the edge of an upright cylinder, again in a direction opposite to true rotation (Fig. 5C).

$<$ Insert Figure 5 about here $>$

Our results are consistent with the hypothesis that the vestibular system resolves ambiguous linear acceleration information from the otolith afferent information, at least in part, on the basis of the head motion frequency content (Paige 1996). However, frequency segregation does not completely resolve the ambiguity (Wood 2002). First, the phase of the perceived motion does not vary as a function of frequency, as one would predict from high- and low-pass filtering alone. Second, there will be at some point a cross-over of low- and high-pass information where the resolution of tilt and translation information by frequency content is more complex. Several studies have demonstrated that the central nervous system utilizes other sensory systems, e.g., semicircular canals and vision, to resolve the ambiguity of tilt and translational linear acceleration stimuli (Angelaki et al. 2004; Merfeld et al. 2005). Neurophysiological evidence for this stems from the observation that neurons in the vestibular nuclei that respond to tilt or translation often receive canal input (Angelaki and Dickman 2003).

The large majority of previous human OVAR studies have been limited to frequencies below $0.3 \mathrm{~Hz}$. These studies have demonstrated the perception of a conical motion path with the amplitude dependent on the tilt angle (Graybiel and Miller 1970; Denise et al. 1988). Our results were consistent in that the amplitude of tilt perception approximately doubled when moving from $10^{\circ}$ to $20^{\circ}$ tilt angle. Except for some limited recordings using a goggle device by Graybiel and Miller (1970), previous studies have relied on verbal reports. During OVAR at high frequency, Miller and Graybiel (1973) reported their subjects had the sensation of "being at or near upright." However, there was no indication whether subjects felt any sense of translation. Noteworthy in the present study is that the perception of reduced tilt at the high frequency is accompanied with increased translation. 
The effects of stimulus frequency on perceived tilt amplitude are not specific to OVAR, but should be present in other motion paradigms involving linear acceleration without visual and canal inputs. For example, similar results have been demonstrated during variable radius centrifugation and/or during translations along a linear track (Glasauer 1995; Merfeld et al. 2005). Although perception of translation has never been reported in earlier studies, the modulation of horizontal SPV during OVAR has been observed by numerous investigations (Guedry 1965; Benson and Bodin 1966; Correia and Money 1970; Young and Henn 1975; Raphan et al. 1981; Cohen et al. 1983; Hain 1986; Wall and Furman 1989; Furman et al. 1992; Clément et al. 1995; Angelaki and Hess 1996). It is generally accepted that this modulation of horizontal SPV represents a translational otolith-ocular response to the modulation of interaural acceleration generated during OVAR (Angelaki and Hess 1996). Therefore, the increase in the amplitude of translation perception as a function of stimulus frequency is consistent with increasing amplitude of translational ocular reflexes.

Testing otolith responses induced by both low- and high-frequency linear accelerations is of interest for assessing vestibular disorders (Furman et al. 1992) or astronauts returning from spaceflight (Clément et al. 1995). One advantage of OVAR over other types of linear acceleration paradigms is that the amplitude of linear acceleration can be precisely maintained across a large frequency range, including low frequencies. For example, linear acceleration of $0.34 \mathrm{~g}$ at $0.125 \mathrm{~Hz}$, such as used in the present study during OVAR at $20^{\circ}$ tilt angle, would require a 5.4-m long linear sled. Variable radius centrifugation has been used to overcome the limitations of track length that often preclude the investigation of low frequency linear acceleration using linear sleds (Glasauer 1995; Seidman et al. 1998; Merfeld et al. 2005). Unfortunately, the linear coriolis forces during variable radius centrifuge become greater at higher frequencies. Although OVAR has been used to test motion sickness sensitivity, higher frequency OVAR has the advantage of being less provocative (Denise et al. 1996; Wood 2002). Perception of translation has not been studied during high frequency linear acceleration, although there is a lot of high frequency content during normal activities (MacDougall and Moore 2005). One could argue that testing at high frequency would be more relevant for evaluating functional deficits in dynamic vestibular responses (Curthoys 2000).

One implication of our findings is that techniques that rely on visual references for selfmotion reports during linear acceleration stimuli are likely to be confounded because of the 
underlying differences in phase between eye movements and perception as a function of stimulus frequency. Wade and Curthoys (1997) have shown the influence of torsion on the amplitude of subjective visual vertical during static tilts. During dynamic linear stimuli, similar effects on the phase of visual vertical would be expected as a function of frequency. Similarly, visual psychophysical measures to determine perceived translation would also be confounded by highpass filtering of horizontal eye movements, especially at frequencies below $0.3 \mathrm{~Hz}$ where there are large phase leads (Wood 2002; Merfeld et al. 2005). Nevertheless, translation perception reporting such as the one in this study is a complementary technique to eye measurement recording that will provide additional information on the cognitive processing of high-frequency otolith information.

\section{REFERENCES}

Angelaki DE, Dickman JD (2003) Gravity or translation: central processing of vestibular signals to detect motion or tilt. J Vestib Res 13: 245-253

Angelaki DE, Hess BJ (1996) Three-dimensional organization of otolith-ocular reflexes in rhesus monkeys. I. Linear acceleration responses during off-vertical axis rotation. J Neurophysiol 75: 2405-2424

Angelaki DE, Shaikh AG, Green AM, Dickman JD (2004) Neurons compute internal models of the physical laws of motion. Nature 430: 560-564

Benson AJ, Bodin MA (1966) Interaction of linear and angular accelerations on vestibular receptors in man. Aerosp Med 37: 144-154

Clément G, Darlot C, Petropoulos A, Berthoz A (1995) Eye movements and motion perception induced by off-vertical axis rotation (OVAR) at small angles of tilt after spaceflight. Acta Otolaryngol (Stockh) 115: 603-609

Cohen B, Suzuki JI, Raphan T (1983) Role of the otolith organs in generation of horizontal nystagmus: effects of selective labyrinthine lesions. Brain Res 276: 159-164

Correia MJ, Money KE (1970) The effect of blockage of all six semicircular canal ducts on nystagmus produced by dynamic linear acceleration in the cat. Acta Oto-Laryngologica 69: $7-16$

Curthoys IS (2000) Vestibular compensation and substitution. Curr Opin Neurol 13: 27-30 
Curthoys IS, Blanks RH, Markham CH (1977) Semicircular canal functional anatomy in cat, guinea pig and man. Acta Otolaryngol (Stockh) 83: 258-265

Darlot C, Denise P, Droulez J, Cohen B, Berthoz A (1988) Eye movements induced by offvertical axis rotation (OVAR) at small angles of tilt. Exp Brain Res 73: 91-105

Denise P, Darlot C, Droulez J, Cohen B, Berthoz A (1988) Motion perceptions induced by offvertical axis rotation (OVAR) at small angles of tilt. Exp Brain Res 73: 106-114

Denise P, Etard O, Zupan L, Darlot C (1996) Motion sickness during off-vertical axis rotation: prediction by a model of sensory interactions and correlation with other forms of motion sickness. Neurosci Lett 203: 183-186

Engelken EJ, Stevens KW (1990) A new approach to the analysis of nystagmus: an application for order-statistic filters. Aviation Space \& Environmental Medicine 61: 859-864

Furman JM, Schor RH, Schumann TL (1992) Off-vertical axis rotation: a test of the otolithocular reflex. Ann Otol Rhinol Laryngol 101: 643-650

Glasauer S (1995) Linear acceleration perception: frequency dependence of the hilltop illusion. Acta Otolaryngol Suppl 520: 37-40.

Graybiel A, Miller EFI (1970) The otolith organs as a primary etiological factor in motion sickness: with a note on "off-vertical" rotation. In: Fourth Symposium on the Role of the Vestibular Organs in Space Exploration, vol SP-187. NASA, Naval Aerospace Medical Research Laboratory; Pensacola, FL, pp 53-66

Guedry FE, Jr. (1965) Orientation of the rotation-axis relative to gravity: its influence on nystagmus and the sensation of rotation. Acta Otolaryngol (Stockh) 60: 30-48

Hain TC (1986) A model of the nystagmus induced by off vertical axis rotation. Biological Cybernetics 54: 337-350

Haslwanter T, Jaeger R, Mayr S, Fetter M (2000) Three-dimensional eye-movement responses to off-vertical axis rotations in humans. Exp Brain Res 134: 96-106.

MacDougall HG, Moore ST (2005) Marching to the beat of the same drummer: the spontaneous tempo of human locomotion. J Appl Physiol 99: 1164-1173

Mayne R (1974) A systems concept of the vestibular organs. In: Kornhuber HH (ed) Handbook of Sensory Physiology, vol VI/2. Springer Verlag, Berlin Heidelberg New York, pp 493580 
Merfeld DM, Park S, Gianna-Poulin C, Black FO, Wood S (2005) Vestibular perception and action employ qualitatively different mechanisms. I. Frequency response of VOR and perceptual responses during Translation and Tilt. J Neurophysiol 94: 186-198

Miller EFI, Graybiel A (1973) Perception of the upright and susceptibility to motion sickness as functions of angle of tilt and angular velocity in off-vertical rotation. In: Fifth Symposium on the Role of the Vestibular Organs in Space Exploration, vol SP-314. NASA, Naval Aerospace Medical Research Laboratory; Pensacola, FL, pp 99-103

Paige GD (1996) How does the linear vestibulo-ocular reflex compare with the angular vestibulo-ocular reflex? In: Baloh RW, Halmagyi GM (eds) Disorders of the Vestibular System. Oxford University Press, New York, pp 93-104

Raphan T, Cohen B, Henn V (1981) Effects of gravity on rotatory nystagmus in monkeys. Annals of the New York Academy of Sciences 374: 44-55

Seidman SH, Telford L, Paige GD (1998) Tilt perception during dynamic linear acceleration. Exp Brain Res 119: 307-314

Wade SW, Curthoys IS (1997) The effect of ocular torsional position on perception of the rolltilt of visual stimuli. Vision Res 37: 1071-1078

Wall Cd, Furman JM (1989) Nystagmus responses in a group of normal humans during earthhorizontal axis rotation. Acta Oto-Laryngologica 108: 327-335

Wood SJ (2002) Human otolith-ocular reflexes during off-vertical axis rotation: Effect of frequency on tilt-translation ambiguity and motion sickness. Neurosci Lett 323: 41-44

Wood SJ, Paloski WH, Reschke MF (1998) Spatial coding of eye movements relative to perceived head and earth orientations during static roll-tilt. Exp Brain Res 121: 51-58

Yagi T, Kamura E, Shitara A (2000) Three dimensional eye movement analysis during off vertical axis rotation in human subjects. Arch Ital Biol 138: 39-47

Young LR, Henn VS (1975) Nystagmus produced by pitch and yaw rotation of monkeys about non-vertical axes. Fortschr Zool 23: 235-246 
Table 1: Verbal reports of motion path. The asteriks indicate those subjects who did complete the entire experiment protocol with both directions of rotation. The responses for these subjects were consistent for both directions of stimulation.

\begin{tabular}{c|c|c|c} 
Subjects & $10^{\circ} @ 0.125 \mathrm{~Hz}$ & $20^{\circ} @ 0.125 \mathrm{~Hz}$ & $20^{\circ} @ 0.5 \mathrm{~Hz}$ \\
\hline 1 & cone & cylinder & cylinder \\
$2^{*}$ & cone & cone & cylinder \\
$3^{*}$ & cone & cone & tight cone \\
$4 *$ & cone & cone & cylinder \\
$5^{*}$ & cone & cone & cylinder \\
6 & cone & cone & cylinder \\
7 & cone & cone & tight cone \\
8 & cone & cylinder & not recorded \\
$9 *$ & cone & cone & cylinder \\
10 & cone & cylinder & cylinder \\
11 & cone & cone & not recorded \\
$12^{*}$ & cone & cone & cylinder \\
13 & cone & cone & not recorded \\
$14 *$ & cone & cone & cylinder
\end{tabular}

\section{FIGURE CAPTIONS}

Fig. 1 Illustration of joystick displacement used to indicate the perception of tilt and conical motion path (A) or translation and cylindrical motion path (B).

Fig. 2 Representative ocular (torsion, horizontal SPV) and perceptive (roll and pitch tilt, ML and AP translation) responses from one subject during OVAR at both $0.125 \mathrm{~Hz}(\mathrm{~A})$ and $0.5 \mathrm{~Hz}(\mathrm{~B})$.in the $\mathrm{CW}$ direction. Note the reduction in both torsion and tilt perception at $0.5 \mathrm{~Hz}(\mathrm{~B})$ relative to $0.125 \mathrm{~Hz}$ (A). The modulation of horizontal SPV and translation perception shows the opposite trend, increasing with stimulus frequency. 
Fig. 3 A. The average modulation amplitude ( \pm sem) for the ocular responses at each of the three stimulus conditions. Note that the amplitude of torsion (solid circles) is indicated by the left ordinate axis, while the horizontal SPV amplitude (open circles) is indicated by the right ordinate axis. Significant differences $(\mathrm{p}<0.05)$ between $10^{\circ}$ and $20^{\circ} \mathrm{OVAR}$ at $0.125 \mathrm{~Hz}$ is indicated by '*' and significant differences $(\mathrm{p}<0.05)$ between $0.125 \mathrm{~Hz}$ and $0.5 \mathrm{~Hz}$ at $20^{\circ} \mathrm{OVAR}$ is indicated with '**.' B. The average phase $( \pm \mathrm{sem})$ for the ocular responses at each of the three stimulus conditions. Note that the average torsion phase (solid circles) was lagging for all conditions, and is indicated with increasing phase lag in the upward direction indicated by the left ordinate axis. The horizontal SPV phase (open circles) was leading in all conditions, and is shown with increasing phase lead in the upward direction indicated by the right ordinate axis. Note there was a significant effect of frequency as indicated by the apparent cross-over in both amplitude and phase. Significant differences are shown as in A.

Fig. 4 A, B. The average ( \pm sem) roll tilt (solid circles) and medial-lateral (ML, open circles) translation perception amplitude and phase at each of the three stimulus conditions. C, D. The average $( \pm$ sem) pitch tilt (solid circles) and anterior-posterior (AP, open circles) translation perception amplitude and phase at each of the three stimulus conditions. Significant differences are shown as in Fig. 3.

Fig. 5 A. The change in orientation during CW OVAR from nose-down (ND), right-ear-down (RED), nose-up (NU) and left-ear-down (LED). B. This sequence of orientation will result in the sensation of tilt following a conical motion path in a CCW direction during rotation at low frequency (Adapted from Denise et al. 1988). C. At high frequency of rotation, it will result in the sensation of translating along a cylindrical motion path in a $\mathrm{CCW}$ direction. 
Figure 1

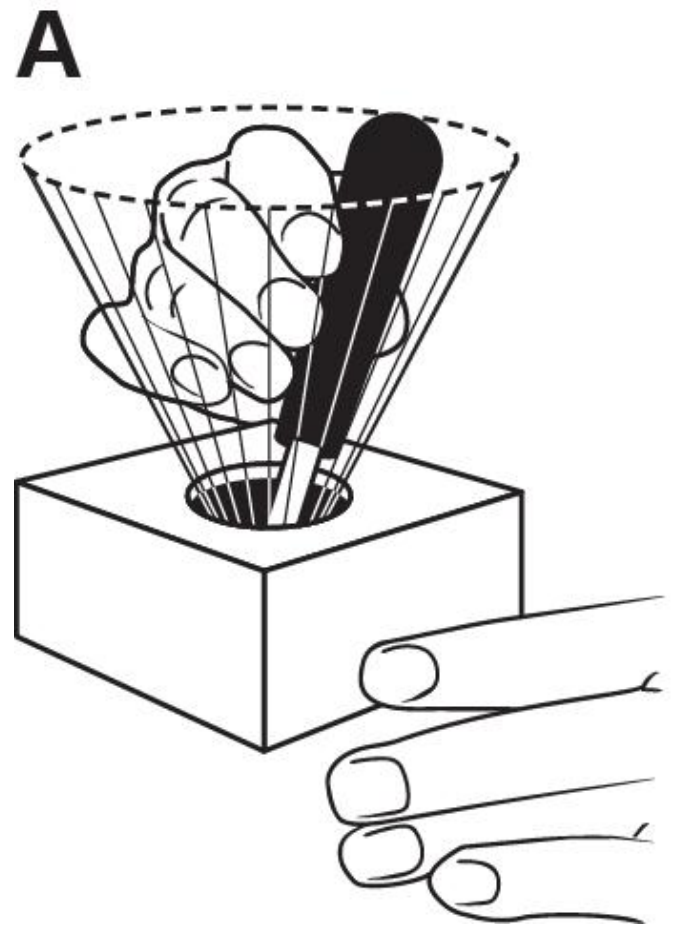

B

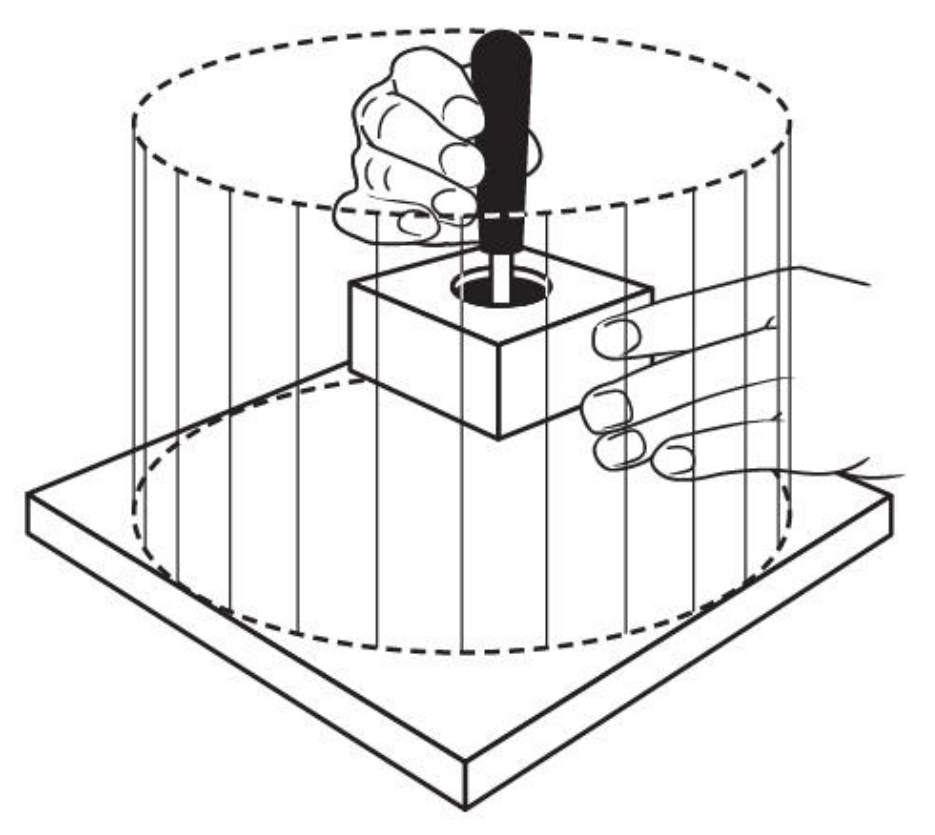


Figure 2

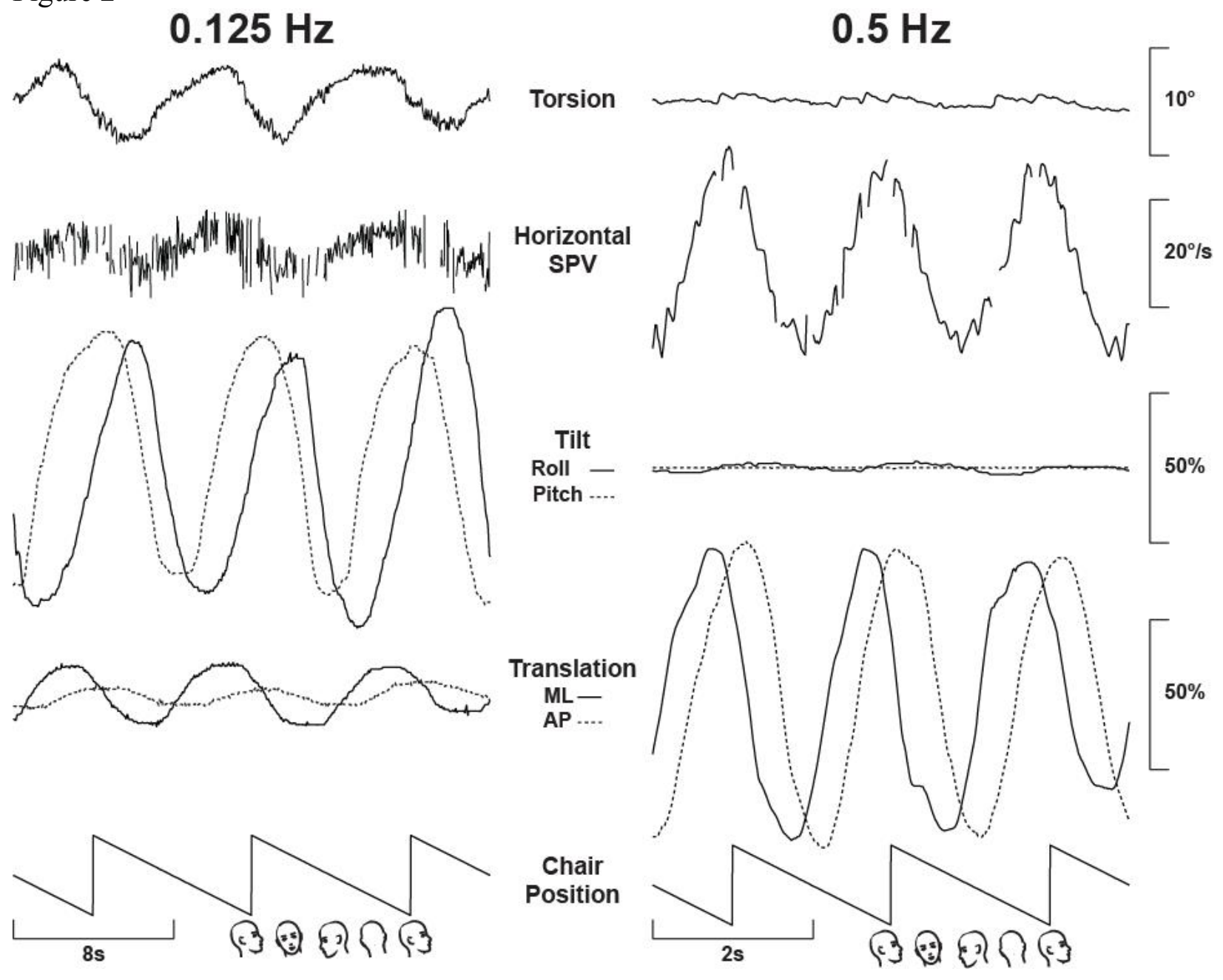


Figure 3

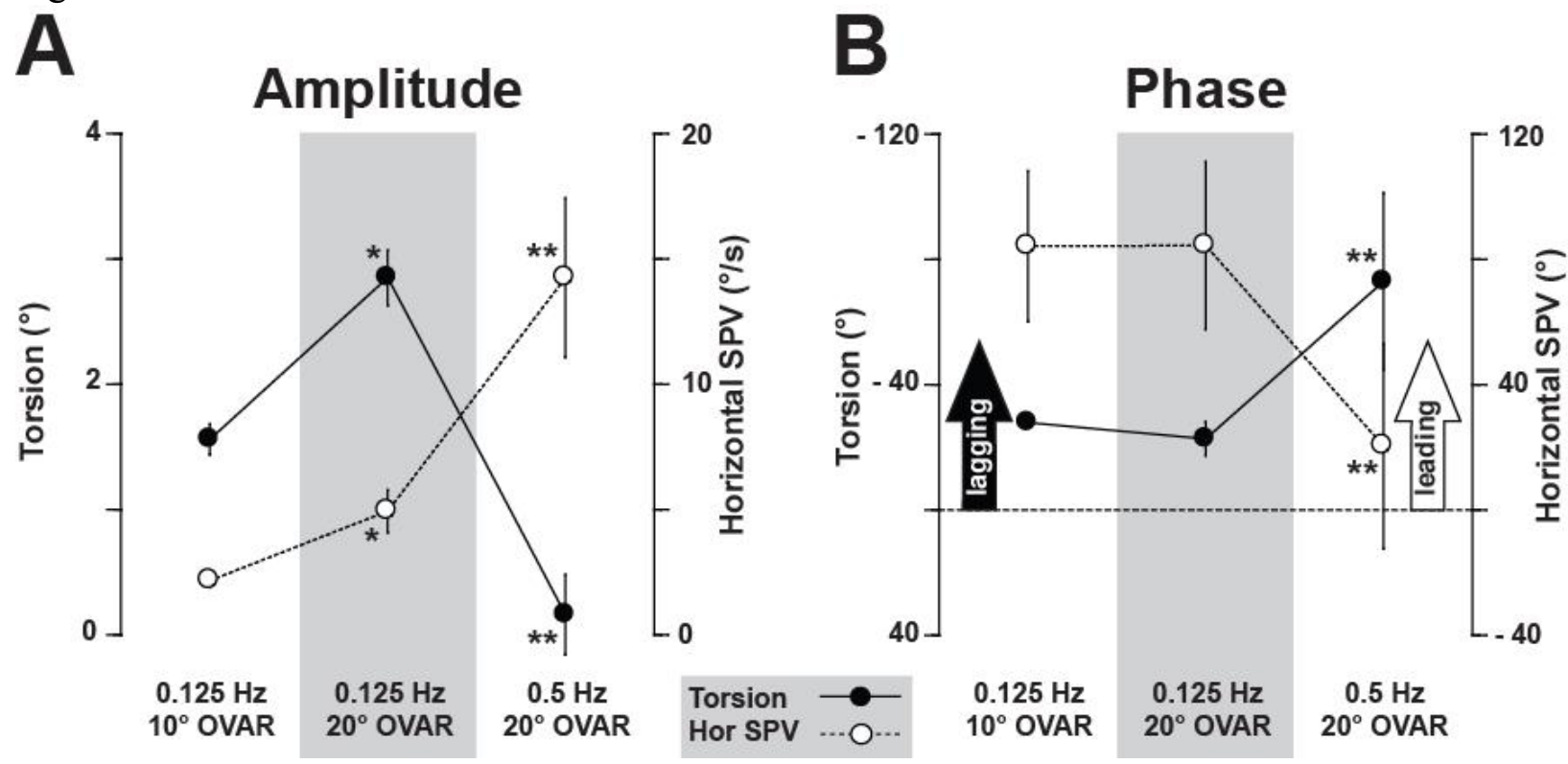


Figure 4

A

B
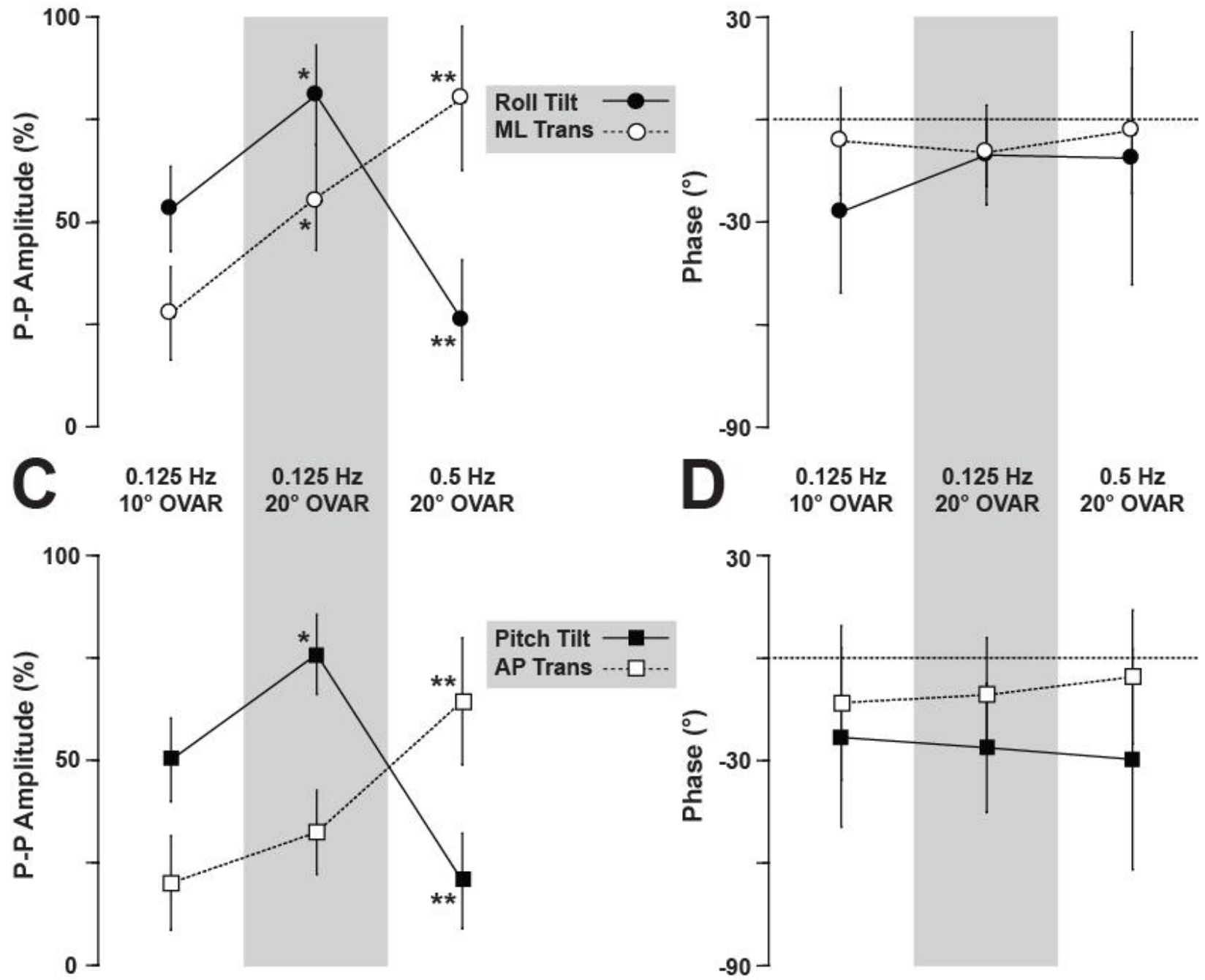

$\begin{array}{cccc}0.125 \mathrm{~Hz} & 0.125 \mathrm{~Hz} & 0.5 \mathrm{~Hz} \\ 10^{\circ} \text { OVAR } & 20^{\circ} \text { OVAR } & 20^{\circ} \text { OVAR }\end{array}$

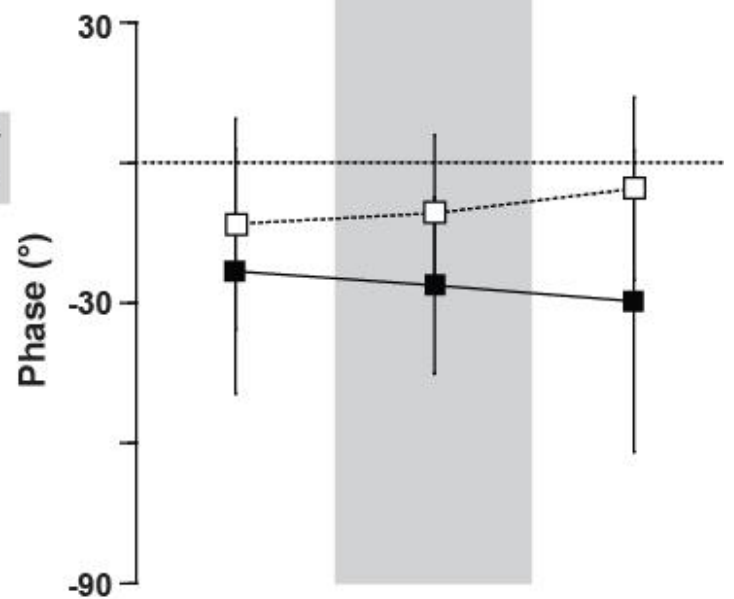


Figure 5

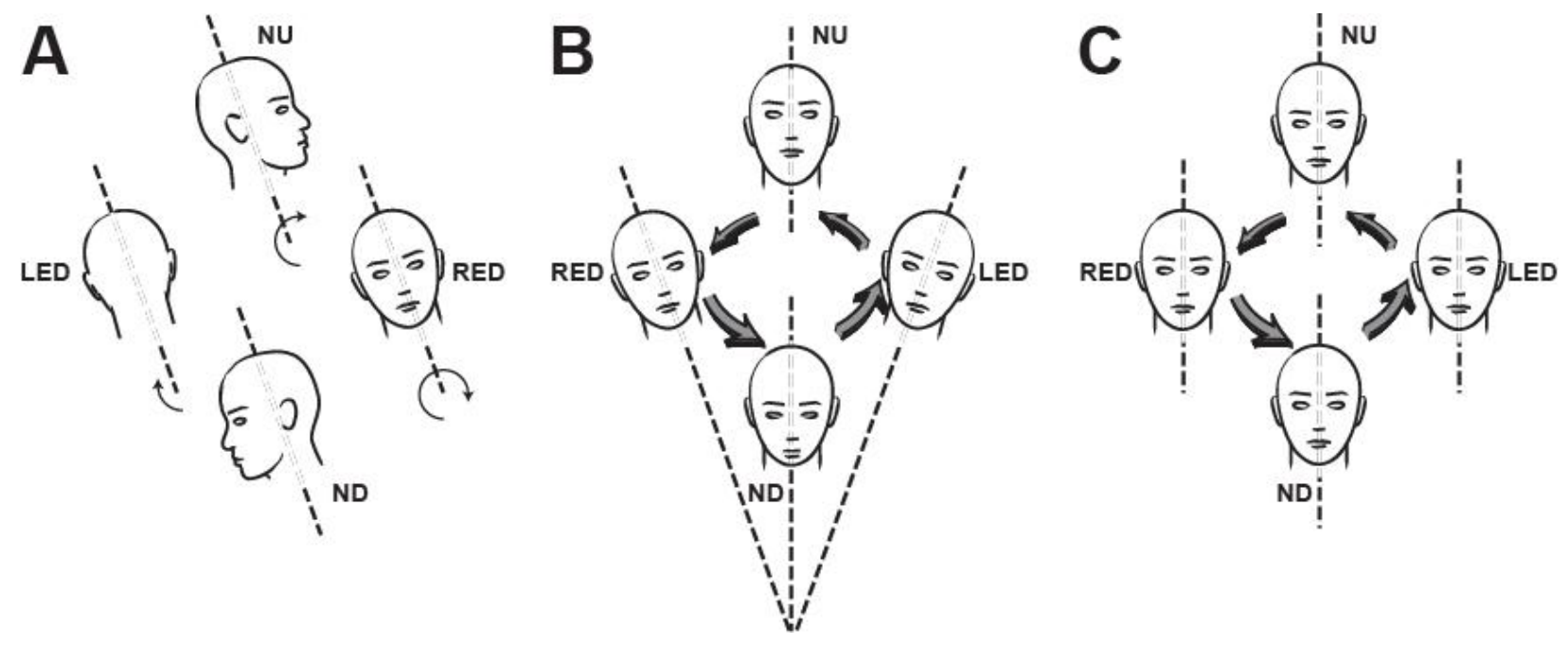

